\title{
Erratum: Evaluation of Semantic Term and Gene Similarity Measures
}

\author{
Michal Kozielski and Aleksandra Gruca \\ Silesian University of Technology, Akademicka 16, 44-100 Gliwice, Poland \\ \{michal.kozielski, aleksandra.gruca\}@polsl.pl
}

S.O. Kuznetsov et al. (Eds.): PReMI 2011, LNCS 6744, pp. 406-411, 2011.

(C) Springer-Verlag Berlin Heidelberg 2011

DOI 10.1007/978-3-642-21786-9_76

By mistake, the following funding information was not included in the original version of the paper:

Acknowledgments. This paper was partially supported by the European Community through the European Social Fund. 